\title{
Trauma and Sharp tooth induced labial vestibule hematoma in a patient with elevated International normalized ratio.
}

\author{
${ }^{1}$ Dr Ajay Kumar Pillai., ${ }^{2}$ Dr J. Arun Kumar., ${ }^{3}$ Dr Swapnil Moghe., \\ ${ }^{4}$ Dr Vineesh Vishnu., ${ }^{5}$ Dr Sakshamnahar, ${ }^{6}$ Dr SaurabhKale. \\ ${ }^{1}$ Reader,Dept of Maxillo Facial Surgery.PDA.PeoplesUniversity.Bhopal \\ ${ }^{2}$ Reader. Dept of Maxillo Facial Surgery.VMS Dental College.Salem \\ ${ }^{3}$ Dept of Maxillo Facial Surgery.PDA.PeoplesUniversity.Bhopal. \\ ${ }^{4} P G$, dept of Community Medicine. PDA.PeoplesUniversity.Bhopal \\ ${ }^{5}$ PG. Dept of Maxillo Facial Surgery.PDA.PeoplesUniversity.Bhopal. \\ ${ }^{6}$ PG.Dept of Pedodontia.PDA.PeoplesUniversity.Bhopal
}

\begin{abstract}
Summary: Haemostasis is a defence mechanism that protects vascular integrity, avoids blood loss, and maintains blood fluidity throughout the circulatory system. Per oral hematoma secondary to anticoagulation is a rare fatal condition. Haemorrhagic complications of warfarin therapy are well known.In any case, the most important concern is the prevention of bleeding complications by compiling a detailed clinical history, with adequate planning of treatment, and taking special care to avoid soft tissue damage during the dental treatment of such patients. Only occasional reports state sublingual and labial vestibule hematoma formation as a result of deficient coagulation. This particular case is very unique in that the patient was on warfarin for the past 3 years but did not develop any hematoma. However trauma by fall triggered the episode of sublingual and buccal vestibular hematoma in this patient. The patient was managed by temporarily stopping warfarin for 4 days and transfusing 2 units of fresh frozen plasma. Tab. Warfarin was restarted after 4 days on alternate days and INR dropped to 2. The sharp cusp of the mandibular tooth was smoothened to prevent ulcer development. The hematoma resolved and no new hematoma formation was observed for a period of 6 months.
\end{abstract}

Key Words: blood coagulation/physiology; blood coagulation disorders/complications; dental care, Haemostasis.

\section{Introduction:}

Hematoma formation secondary to anticoagulation is a rare but a possible potentially fatal complication.Hemorrhage is the principle adverse effect of oral anticoagulants. Spontaneous airway threatening bleeding has been reported in the sublingual and retropharyngeal tissue spaces in patients whose anticoagulation levels are poorly controlled and above the expected therapeutic levels. Early diagnosis and immediate intervention is mandatory in such conditions.

There are several currently recognized indications for the use of warfarin. These include deep

venous thrombosis, pulmonary embolism, vascular thrombo-embolism, transient cerebral ischemic attack, stroke and prosthetic valve replacement. The coagutherapy level is measured in values of the International Normalized Ratio (INR)1. Warfarin dosages are adjusted to achieve an optimum therapeutic level of anticoagulation, and this is best achieve by monitoring prothrombin time being expressed as INR ratio with the therapeutic levels accepted between $2-4\{1\}$. This particular case is very unique in that the patient was on warfarin for the past 3 years but did not develop per oral hematoma. However trauma by an attrited sharp cusp/ trauma due to fall triggered the episode of sublingual and labial vest hematoma. The presentation of this case makes dental examination for sharp cusps mandatory for all patients who will be administered warfarin.

\section{Case Presentation}

A 48 year old lady reported to the clinic complaining about a painful swelling in the floor of the mouth and in lower lips. The pain was sudden in onset, intermittent, dull aching type, non-radiating, aggravated on swallowing food and was present for 2 days. The swelling was preceded by an accidental trauma to floor of the mouth while she fell down. It was also sudden in onset and progressed to the present size. The swelling elevated the tongue causing dysphagia but did not cause airway obstruction. There was noticeable swelling in lower lip.

Patient's medical history revealed mitral valve stenosis for which mitral valve replacement was done 3 years back. Her drug history revealed that she was on Tab. Ecosprin $75 \mathrm{mg}$ and Tab. Warfarin $7.5 \mathrm{mg}$ OD. Dental history was non-contributory. 
The vital signs revealed pulse rate of $76 / \mathrm{min}$ and $\mathrm{BP}$ of $92 / 68 \mathrm{mmHg}$. Intraoral examination revealed a solitary swelling measuring about $2.5 \times 3.5 \mathrm{~cm}$ in the floor of the mouth extending anteroposteriorly from the alveololingual sulcus, to the junction between the floor of the mouth and base of the tongue. The swelling also extended bilaterally to both right and left lingual and labial sulci[figure 1]. The mucosa over the swelling was erythematous. Bluish discoloration was present on the tip of the tongue,labial mucosa and the swelling displaced the tongue superiorly. The swelling, on palpation, was soft in consistency, tender, and non-pulsatile.

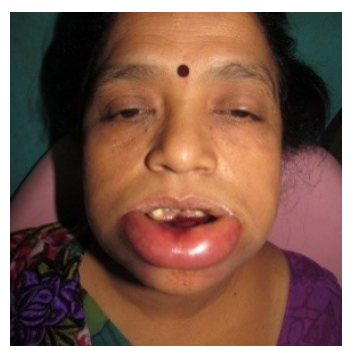

Figure 1:frontal profile of the patient.

Based on the clinical finding, a provisional diagnosis of sublingual and lower labial vestibule hematoma was considered.

\section{Investigations}

- OPG (Figure -2) revealed no significant findings.

- Blood investigations revealed all parameters were within normal limits. However there was elevated International Normalised Ratio (INR) of 13.5

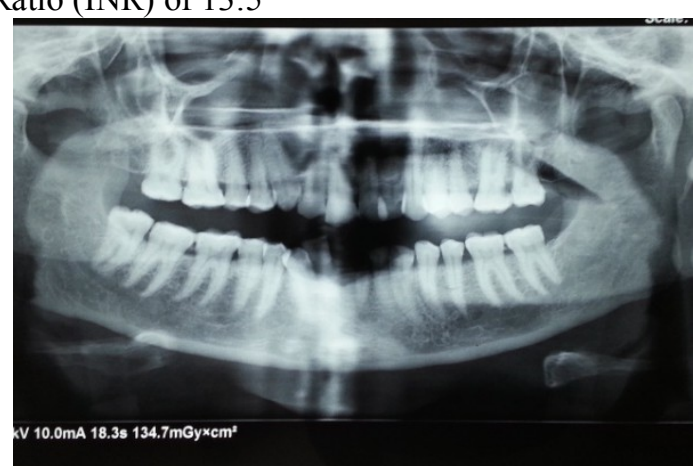

Figure 2: OPG

\section{Differential Diagnosis}

The history, clinical findings and investigations suggesttrauma andsharp tooth induced sublingual and lower labial vestibule hematoma in a patient with elevated international normalized ratio. Fracture of the mandible can also present with sublingual hematoma and it was ruled out in this patient.

\section{Treatment}

After taking physician opinion,the patient was managed by temporarily stopping warfarin for 4 days and transfusing 2 units of fresh frozen plasma after obtaining physician opinion. Tab. Warfarin was restarted after 4 days on alternate days and INR dropped to 2 .

The hematoma gradually resolved over a period of few days. Follow up was done for 6 months and no new hematoma formation was observed during this period (Figure - 3).

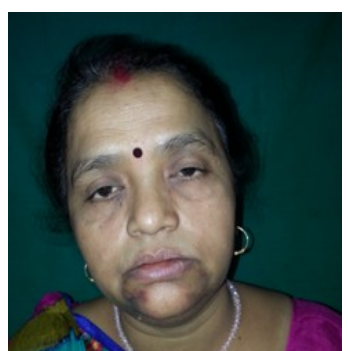

Figure 3: Post operative Follow up after two weeks. 


\section{Discussion}

Warfarin therapy reduces the risk of thromboembolic events. The antithrombotic effect of warfarin is the result of its interference with the hepatic synthesis of vitamin $\mathrm{K}$ dependent clotting factors II, VII, IX and X. In addition, it interferes with the synthesis of the anticoagulant proteins $\mathrm{C}$ and S.[1,2]

The management of oral surgery procedures on patients treated with anticoagulants should be influenced by several factors which include physician's recommendation, dentist expertise extent and urgency of surgery, laboratory values, available facilities,and patient's oral, medical, and general condition.[1]

The major challenge in warfarin therapy is its narrow therapeutic range. Even a mild degree of over anticoagulation may lead to haemorrhage. Bleeding is the most serious complication of warfarin therapy.[2,3,4,5] Estimated annual incidences of bleeding rates are $0.6 \%$ for fatal bleeding, $3.0 \%$ for major bleeding, and $9.6 \%$ for minor bleeding.[6,7,8] In addition to sublingual region,, haemorrhages do occur in other parts of the body such as the genitourinary, gastrointestinal, retroperitoneal and intracranial regions.[9] Traditionally this type of situation was managed by interruption of anticoagulant therapy there by putting the patient at risk of a potentially life-threatening thromboembolic crisis. It has been reported that if the anticoagulant therapy is stopped is three to five times greater than the risk of the patient having postoperative bleeding that cannot be controlled with local measures.

Indications for the use of oral anticoagulants includes mechanical valve replacement for Rheumatic heart disease, deep venous thrombosis, pulmonary embolism, vascular thromboembolism, cerebral venous thrombosis, certain hypercoaguable states, and transient ischemic attack.[10]

As per the literature search the author feel the need need to make a protocol for oral surgery procedures in patients on oral anticoagulant therapy. As per the literature, the Author proposes following measures to be taken.

1. All patients should have a preoperative INR measured and this should be in the range of 2-4.

2. Patients should not have other major risk factors or taking medications affecting the liver or renal function.

3. The use of good surgical technique and local measure consisting of absorbable oxidized cellulose pack and careful suturing should be employed in all patients.

4. A spontaneous history of nasal or oral bleeding of such patients should be noted.

5. The presentation of this case makes dental examination for sharp cusps mandatory for all patients who will be administered warfarin.

6. The dental examination for sharp cusps mandatory for all patients who will be administered warfarin

In the present case, sublingual and buccal vestibule hematoma developed due to elevated INR of 11.2. However, a sharp cusp in the left mandibular tooth traumatized the labial vestibule of the mouth and exaggerated the condition during functional movements. A minority of cases in the literature have indicated a causative factor for sublingual hematoma arising from trauma alone without any anticoagulation significance, such as after oral surgical procedures.[11]

In those surgical extractions which may require extensive flaps and substantial bone removal, where higher risk of postoperative bleeding is expected, modifications in the warfarin dosage might be considered in liaison with the patient's treating physician, which undoubtedly should not be simple discontinuation of the patient anticoagulation therapy, as there are few alternatives such as switching to heparin or low-molecularweight heparins.[1] After obtaining physician recommendation, Warfarin in our case was temporarily withheld and fresh frozen plasma was administered for immediate reversal of anticoagulation effects of Warfarin. Warfarin was restarted after 4 days on alternate days and INR dropped to 2. Smoothening of the sharp cusp in the tooth prevented trauma to the floor of the mouth and averts hematoma formation in future.

Fresh frozen plasma is less expensive than prothrombin complex concentrate and has reduced risk of thromboembolism. Fresh frozen plasma contains all vitamin K-dependent factors and is often used in conjunction with vitamin $\mathrm{K}$ and prothrombin complex concentrates. However, one significant side effect that remains is the volume-associated sequelae from fluid overload, suffered as a result of infusion. It also needs to be group specific. Additionally, FFP is the most commonly blamed blood product in transfusion-related acute lung injury (TRALI), and the risks and benefits of transfusion must be carefully weighed in each patient. The volume of PCC needed to reverse anticoagulation is far less than the volume of FFP that is needed (typically only about 5\%), which decreases the risk of fluid overload. Furthermore, infusion of FFP has been known to increase the risk of TRALI, a major cause of mortality associated with transfusion. No incidents of TRALI following administration of PCCs have been reported. However clinicians continue to use FFP because of the lower cost per unit.[12]

It is also worth acknowledging the necessity of surgical drainage versus non-surgery in the case of management of the sublingual hematoma. If there is any indication of severe obstruction or edema within the pharynx or larynx during the endoscopic examination no hesitation should be made to establish a surgical 
airway immediately. Spontaneous resolution of the hematoma once coagulation is normalized occurs in most cases. [13] In our patient however, since the hematoma resolved, surgical decompression was not required.

\section{References}

[1]. Medyan al-rousan.dental management of patients on warfarin therapy.Oral medicine/oral \& maxillofacial surgery.Pakistan Oral \& Dental Journal Vol 30, No. 1, (June 2010)

[2]. John E. Murphy. Clinical Pharmacokinetics.Ann K. Wittkowsky. Warfarin. American Society of Health-System Pharmacists, Inc.. $5^{\text {th }}$ edition. 2012. p 351

[3]. Schulman S, Beyth RJ, Kearon C, et al. Hemorrhagic complications of anticoagulant treatment: American College of Chest Physicians Evidence-Based Clinical Practice Guidelines (8th Edition). Chest 2008;133:257S-298S.

[4]. Beyth RJ. Hemorrhagic Complications of oral anticoagulant therapy.ClinGeriatr Med. 2001;17(1): 49-56.

[5]. Dahri K, Loewen P. The risk of bleeding with warfarin: A systematic review and performance analysis of clinical prediction rules. ThrombHaemost. 2007;98:980-987.

[6]. Landefeld CS, Beyth RJ. Anticoagulant-related bleeding: clinical epidemiology, prediction, and prevention. AM J Med. 1993;95:315-28.

[7]. Dahri K, Loewen P. The risk of bleeding with warfarin: A systematic review and performance analysis of clinical prediction rules. ThrombHaemost. 2007;98:980-987.

[8]. Landefeld CS, Beyth RJ. Anticoagulant-related bleeding: clinical epidemiology, prediction, and prevention. AM J Med. $1993 ; 95: 315-28$

[9]. González-García R, Schoendorff G, Muñoz-Guerra MF, Rodríguez-Campo FJ, et al. Upper airway obstruction by sublingual hematoma: a complication of anticoagulation therapy with acenocoumarol. Am J Otolaryngol 2006;27:129-132.

[10]. AnchalPuri, Muzzammil A Nusrath, Deepak Harinathan, Jamie Lyall. Massive sublingual hematoma secondary to anticoagulant therapy complicated by a traumatic denture: a case report. J Med Case Reports. 2012;6:105.

[11]. Pigadas N, Simoes P, Tuffin JR: Massive sublingual haematoma following osseointegrated implant placement in the anterior mandible. Br Dent J 2009;206:67-68.

[12]. Vigue B. Bench-to-bedside review: Optimising emergency reversal of vitamin K antagonists in severe haemorrhage--from theory to practice. Crit Care. 2009;13:209.

[13]. Pigadas N, Simoes P, Tuffin JR: Massive sublingual haematoma following osseointegrated implant placement in the anterior mandible. Br Dent J 2009;206:67-68. 